\title{
About the dependency of the spin maxima on orbital phase in the intermediate polar MU Cam
}

\author{
Š. Parimucha ${ }^{1}$, P.A. Dubovsky ${ }^{2}$, I. Kudzej ${ }^{2}$, V. Breus ${ }^{3}$ and \\ K. Petrik ${ }^{4,5}$ \\ 1 Institute of Physics, Faculty of Science, UPJS Kosice, Slovakia \\ 2 Vihorlat Astronomical Observatory, Mierova 4, SK-06601 Humenne, Slovak \\ Republic (E-mail:var@kozmos.sk) \\ 3 Department of Mathematics, Physics and Astronomy, Odessa National \\ Maritime University, Mechnikova 34, UA-65029 Odessa, Ukraine (E-mail: \\ bvv_2004@ua.fm) \\ 4 M. R. Štefanik Observatory and Planetarium, Sladkovicova 41, SK-92001 \\ Hlohovec, Slovak Republic \\ 5 Institute of Physics, Faculty of Philosophy 8 Science, Silesian University in \\ Opava, Bezrucovo nam. 13, CZ-746-01 Opava, Czech Republic
}

Received: November 1, 2019; Accepted: January 8, 2020

\begin{abstract}
Long term monitoring of intermediate polars is performed as part of the Inter-Longitude Astronomy campaign. High-quality and long time series observations allow us to investigate fine effects on complex light curves. In the case of MU Cam we have investigated the periodic modulation of the spin phases with the orbital phase. This dependency was already described by Kim et al. (2005). As an explanation they proposed inhomogeneous accretion flow from the secondary. However, based on our new data, we propose as a simple explanation the influence of orbital sidebands in the periodic signal produced by the intermediate polar. This explanation is supported by the fact that the changes in spin maxima phase are observed mainly when the sideband frequency is dominant in the periodogram.

Key words: stars: individual: MU Cam - stars: novae, cataclysmic variables - accretion, accretion discs
\end{abstract}

\section{Observations and data analysis}

MU Cam (1RXS J062518.2+733433) was classified as an intermediate polar by Staude et al. (2003) and Araujo-Betancor et al. (2003). They determined spin and orbital periods of the system.

Our observations of MU Cam were taken as part of the Inter-Longitude Astronomy Campaign (Andronov et al., 2003). The goal of the campaign is the monitoring of selected intermediate polars for spin period changes. In this work we analyze data from the Astronomical Observatory on Kolonica Saddle and 
from M.R. Štefanik Observatory in Hlohovec. We used the Vihorlat National Telescope VNT 1000/9000 mm and Cassegrain 600/2500 mm. Ensemble photometry was performed using C-Munipack, CoLiTecVS (Kudzej et al., 2019) and MCV (Kim et al., 2004) software packages.

We have constructed the O-C diagram of spin pulse maxima based on the ephemeris $B J D_{\max }=2452682.4181+0.01374116815 \times E$. Residuals from the quadratic fit $B J D_{\max }=2452682.4181+0.0137412342412802 \times E-1.52 \times 10^{-12} \times$ $E^{2}$ show unexpected scatter. As a possible source of these deviations we have investigated the dependency of spin maxima timings on orbital phase.

Mean spin maxima were determined for 10 phase intervals: $0.0-0.1 ; 0.1-0.2$, etc. Only long time series were used to achieve higher precision. The amplitude and shape are changing, but there is no unambiguous dependency on orbital phase.

Periodogram analysis reveals two correlations:

1. High amplitude O-C variations of spin maxima - strong sideband signal. Low amplitude - low or no sideband signal.

2. Orbital sideband appears mainly in low states of the long term light curve.

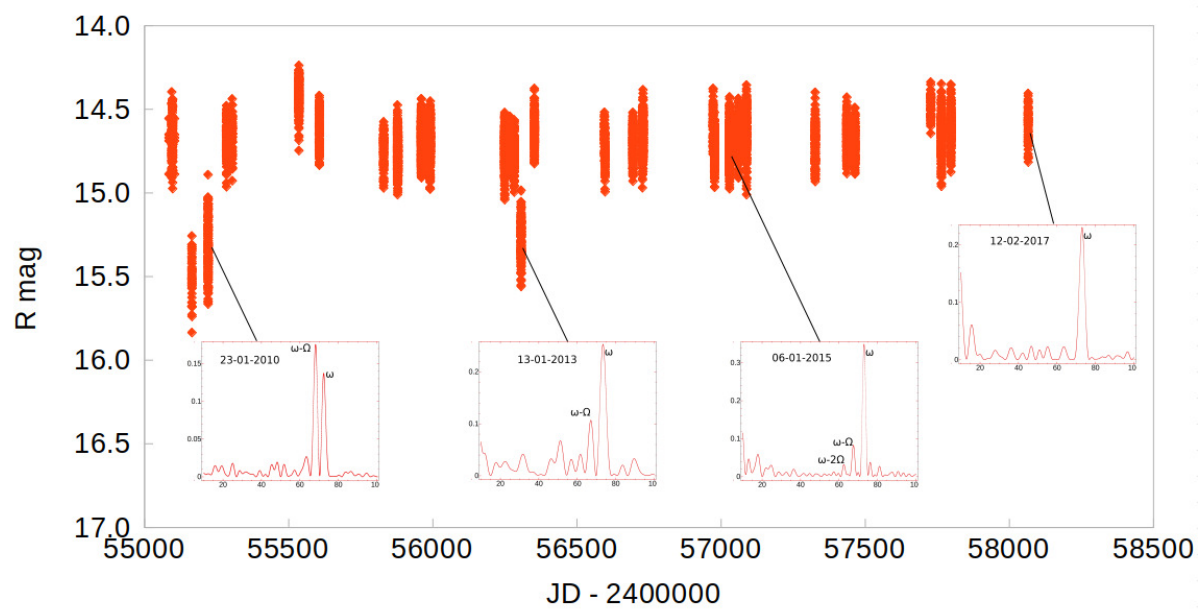

Figure 1. Selected periodograms depicted on the long-term light curve.

\section{Conclusions}

We performed photometric observations of MU Cam, analysed the new and previously published data, and obtained the following results: 
- Spin maxima phase changes are caused by the interaction with the orbital sideband frequencies.

- The presence of orbital sidebands is more prominent in low states.

- The origin of orbital sidebands can be direct accretion from the stream or/and reprocessing of X-rays at some part of the system which rotates with the orbital period.

- The low states in intermediate polars are connected with lower mass transfer in the system. In that situation the disc-fed accretion should be lower. The disc can completely disappear as was demonstrated by Hameury \& Lasota (2017) for the FO Aqr case.

Acknowledgements. This work was supported by the Slovak Research and Development Agency under the contract No. APVV-15-0458. VB acknowledges financial support from the National Scholarship Programme of the Slovak Republic.

\section{References}

Andronov, I. L., Antoniuk, K. A., Augusto, P., et al., Inter-longitude astronomy project: some results and perspectives. 2003, Astronomical and Astrophysical Transactions, 22, 793, DOI: 10.1080/1055679031000124501

Araujo-Betancor, S., Gänsicke, B. T., Hagen, H. J., Rodriguez-Gil, P., \& Engels, D., 1RXS J062518.2+733433: A new intermediate polar. 2003, Astron. Astrophys., 406, 213, DOI: 10.1051/0004-6361:20030787

Hameury, J. M. \& Lasota, J. P., The disappearance and reformation of the accretion disc during a low state of FO Aquarii. 2017, Astron. Astrophys., 606, A7, DOI: 10.1051/0004-6361/201731226

Kim, Y., Andronov, I. L., \& Jeon, Y.-B., CCD Photometry Using Multiple Comparison Stars. 2004, Journal of Astronomy and Space Sciences, 21, 191, DOI: 10.5140/JASS.2004.21.3.191

Kim, Y.-G., Andronov, I. L., Park, S.-S., et al., Two-Color VR CCD Photometry of the Intermediate Polar 1RXS J062518.2+733433. 2005, Journal of Astronomy and Space Sciences, 22, 197, DOI: 10.5140/JASS.2005.22.3.197

Kudzej, I., Savanevych, V. E., Briukhovetskyi, O. B., et al., CoLiTecVS - A new tool for the automated reduction of photometric observations. 2019, Astronomische Nachrichten, 340, 68, DOI: 10.1002/asna.201913562

Staude, A., Schwope, A. D., Krumpe, M., Hambaryan, V., \& Schwarz, R., 1RXS J062518.2+733433: A bright, soft intermediate polar. 2003, Astron. Astrophys., 406, 253, DOI: 10.1051/0004-6361:20030705 